\title{
INSTRUMENTOS DE AVALIAÇÃO DA ANSIEDADE: ACHADOS TEÓRICOS E EMPÍRICOS FOCALIZADOS POR PESQUISAS BRASILEIRAS
}

\author{
Adriana Sant'Ana, Antônio Barbosa Alves Junior, Flávia Maria Ferrari Almeida, Gleice Fabrício \\ Barbudo, Igor Bravo Estácio, Camélia Santina Murgo \\ Universidade do Oeste Paulista - UNOESTE, Faculdade de Medicina, Presidente Prudente, SP. E-mail: \\ camélia@unoeste.br.
}

\begin{abstract}
RESUMO
Nota-se um crescente aumento de pesquisas sobre a incidência de ansiedade na população o que tem intensificado a preocupação de estudiosos sobre o tema. Entre outras questões, discute-se a importância da utilização de instrumentos que permitam a avaliação da ansiedade a fim de que possíveis transtornos sejam diagnosticados. Dessa forma, o presente estudo tem como objetivo verificar a produção cientifica em periódicos nacionais referentes à avaliação da ansiedade. No total foram encontrados 35 artigos que descrevem a utilização da Escala de Ansiedade. Observou-se que o período de publicação de maior número de artigos foi nos anos de 2015 e 2016. Também foi verificado que a ansiedade vem sendo estudada em associação com depressão, desesperança, personalidade, neuroticismo, abuso de drogas, conduta antissocial, transtorno de estresse pós - traumático, sonolência diurna, compulsão alimentar e doenças como: fibromialgia, esclerose e parkinson.. Constatou-se que a Escala Beck foi apontada como o instrumento mais utilizado na avaliação da ansiedade
\end{abstract}

Palavras-chave: Ansiedade. Saúde Mental. Psicopatologia. Escalas Beck. Escalas.

\begin{abstract}
There is a growing increase in research on the incidence of anxiety in the population which has intensified the concern of scholars on the subject. Among other issues, it is discussed the importance of the use of instruments that allow the evaluation of anxiety so that possible disorders are diagnosed. Thus, the present study aims to verify the scientific production in national journals regarding the evaluation of anxiety. In total, 35 articles were found describing the use of the Anxiety Scale. It was also observed that the period of publication of the greatest number of articles was in the years of 2015 and 2016. It was also verified that anxiety has been studied in association with depression, hopelessness, personality, neuroticism, drug abuse, antisocial behavior, Post - traumatic stress disorder, daytime sleepiness, binge eating, and diseases such as fibromyalgia, sclerosis and parkinsonism. The Beck Scale was found to be the most used instrument for assessing anxiety
\end{abstract}

Key words: Anxiety. Mental health. Psychopathology. Beck scales. Scales 


\section{INTRODUÇÃO}

A ansiedade é um sentimento de apreensão que aponta para as pessoas que há um perigo, traduzindo-se em manifestações fisiológicas como agitação, hiperatividade e movimentos precipitados e manifestações cognitivas como atenção e vigilância redobrada, pensamentos e possíveis desgraças. Essas manifestações podem ser passageiras ou permanentes variando sua intensidade a níveis imperceptíveis ou elevados (BATISTA e OLIVEIRA, 2005),

Os critérios diagnósticos marcados na DSM-V abrangem: ansiedade e preocupação excessiva e de difícil controle na maioria dos dias e com duração mínima de seis meses, ocasionando prejuízos no funcionamento da vida diária. O quadro deve conter também três de seis sintomas físicos tais como: inquietação; fatigabilidade; dificuldade de concentração; irritabilidade; tensão muscular ou perturbações do sono. (OLIVEIRA et al 2006).

Nessa direção, Oliveira et al (2006) buscaram explorar a relação entre ansiedade, depressão e desesperança entre grupos de idosos. Participaram do estudo 79 idosos provenientes de centro da terceira idade $(50,6 \% ; n=40)$, de um posto de retirada de medicamentos (38\%; $n=30)$, e de uma instituição asilar $(11,4 \% ; n=9)$. Utilizou-se um questionário para os dados de caracterização dos idosos, bem como as escalas Beck para mensurar sintomas de ansiedade, depressão e desesperança. Assim evidenciando que a relação estatisticamente significativa entre ansiedade, depressão e desesperança nos idosos.

Igualmente, Araújo, Nakano e Gouveia (2009), em seu estudo investigaram sintomas de depressão e ansiedade em dois grupos de presidiários (30 recém-chegados ao presídio e 30 antigos). Utilizou-se um instrumento sócio demográfico e as escalas Beck de depressão e ansiedade para a coleta dos dados. Os resultados mostraram que os detentos dos dois grupos apresentaram, em sua maioria, níveis mínimos de depressão e ansiedade, com maior presença de indicadores nos novatos. Uma relação positiva e significativa foi encontrada entre os níveis de depressão e ansiedade, indicando relação entre as duas sintomatologias.

Ainda sobre investigações referentes ao construto ansiedade, Stall, et al (2015) realizaram uma pesquisa com sessenta pacientes $(n=60)$ diagnosticados com Síndrome de Fibromialgia. Os pacientes foram divididos em três grupos onde o grupo A foi submetido a acupuntura; o grupo $B$ ao método de Rolfing e o grupo $C$ acupuntura associada ao método Rolfing. Os pacientes tratados apenas com acupuntura apresentaram um maior nível de ansiedade. Os instrumentos utilizados nesse estudo foram Questionário de Impacto da Fibromialgia, Escala Verbal Numérica de Dor e Inventários de Ansiedade e de Depressão Beck.

Beigin et al (2015) apresentaram um estudo com o objetivo de identificar fatores psicossociais e emocionais, e a qualidade de vida de pacientes com o desencadeamento e a cronificação da síndrome dolorosa após a laminectomia. Participaram do estudo um grupo teste que já eram diagnosticados com síndrome dolorosa pós-laminectomia ( $n=16)$ e um grupo controle submetido a interconsulta na clinica da dor $(n=15)$. Nesse estudo foram utilizados a Escala visual e o dolorímetro de Fischer, o questionário WHOQOL-Bref e os Inventários Beck de Ansiedade e de Depressão.

O estudo de Guerra, et al (2016) analisou o sono, a qualidade de vida e humor em profissionais de enfermagem em Unidades de Terapia Intensiva Infantil foi realizado com 168 profissionais de enfermagem ( $n=168$ ) que foram distribuídos em turnos da manhã (69), tarde (29) e noite (70). Foram utilizados instrumentos como o questionário de identificação Matutinidade-Vespertinidade, escala de sonolência Epworth, questionário genérico de avaliação de qualidade de vida - SF-36, inventário de depressão de Beck, inventário de ansiedade de Beck, inventário de ansiedade Traço-Estado. 
No ano de 2016, Trocoli e Botelho fizeram estudos sobre a prevalência da depressão e cinesiofobia em pacientes com lombalgia e sua associação com os sintomas da lombalgia, onde foram utilizados 65 pacientes $(n=65)$ divididos em três grupos: orgânicos, orgânicos amplificados e não orgânicos. Foram utilizados instrumentos como o inventário de ansiedade de Beck, inventário de depressão de Beck, escala de cinesiofobia de tampa e foram avaliados de acordo com seu nível de dor pela escala Análogo-Numérica.

Recentemente, Lacerda et al (2017) realizaram uma pesquisa sobre a ansiedade, estresse e depressão de familiares de pacientes com insuficiência cardíaca e identificar a relação entre esses sentimentos com as variáveis sociodemográficas e clínicas. Esse estudo foi composto por 100 familiares $(n=100)$ que foram aplicados em instrumentos como inventários de depressão e ansiedade de Beck, escala de estresse percebido - 10 e teste $t$, Mann-Whitney ou Kruskal-Wallis.

Tendo em vista os estudos até aqui apresentados, nota-se, que tem se mostrado pertinente compreender a ansiedade e suas implicações em diversas situações e contextos. Igualmente faz-se necessário dispor de instrumentos adequados para a avaliação desse construto, o que justifica os objetivos da presente pesquisa, a saber, verificar a produção cientifica dos últimos dez anos em periódicos nacionais sobre a temática em questão

\section{MÉTODO}

Para o levantamento bibliográfico, foram utilizados os descritores instrumentos de avaliação da ansiedade, escalas de ansiedade. A base de dados pesquisada foi a BVS-psi na qual se encontram indexadas as bases PEPSIC e SCIELO. Foram encontrados no total 35 artigos que descrevem a utilização da Escala Beck de Ansiedade como instrumentos de avaliação desse construto. o conteúdo dos artigos selecionados para análise permitiu a elaboração de categorias que serão discutidas a partir das tabelas apresentadas nos resultados. Constituíram-se categorias de análise: período de publicação, periódicos com maior incidência de publicações sobre o tema, objetivos dos artigos, população estudada e construtos correlacionados com ansiedade e instrumentos utilizados para avaliação.

\section{RESULTADOS}

Serão trazidos para esta sessão, os principais achados encontrados na produção cientifica recuperada. Inicialmente são apresentados os dados referentes à periodicidade das publicações. A maior prevalência ocorreu nos anos de 2015 e 2016, A maioria das publicações estão nas revistas Revista Dor (3), Avaliação Psicológica (2), Psicologia em Estudo (2), Revista de Psiquiatria do Rio Grande do Sul (2), Revista da Escola de Enfermagem da USP (2) e Escola de Psicologia (Campinas) (2). Nota-se que muitas publicações são de periódicos da área medica como Psiquiatria, Ginecologia, Pediatria, Cardiologia e Enfermagem.

Também considerou-se importante ressaltar os objetivos dos estudos na intenção de uma maior compreensão acerca das preocupações que as publicações vêm trazendo, bem como para visualizar lacunas. A Tabela 1 configura-se, portanto, como um demonstrativo dos objetivos dos estudos agrupados em categorias. 
Tabela 1. Objetivos das Pesquisas

Objetivos

Verificar associações entre construtos diversos

Verificar associações entre ansiedade e depressão

Avaliar qualidade de vida em associação com ansiedade

Avaliar prevalência de ansiedade em associação com doenças

Incidência de Ansiedade em grupos específico
N 16

9

5

$\mathrm{Na}$ Tabela 2 são apresentados os grupos estudados, constatando-se que a população mais investigada foram mulheres mães ou gestantes (6) e estudantes universitários dentre eles Enfermagem, Psicologia, Medicina e Licenciatura (4). Ainda foi possível perceber a partir da análise da tabela que houve uma população diversificada, abrangendo desde jovens a idosos de ambos os sexos, pessoas enfermas, mulheres gestantes e usuários de drogas.

Tabela 2. População estudada

\begin{tabular}{lc}
\hline População & No de artigos \\
\hline Mulheres Gestantes e Mães & 6 \\
Estudantes de Ensino Superior & 4 \\
Idosos & 2 \\
Pacientes Obesos (as) & 2 \\
Usuários de Substancia Psicoativa & 2 \\
Mulheres acometidas de doenças & 2 \\
Homens detentos & 2 \\
Profissionais de enfermagem & 2 \\
Pacientes com Lombalgia, fibromialgia & 2 \\
\hline
\end{tabular}

Cabe também mencionar outros grupos estudados cuja incidência na literatura não foi tão expressiva coo jovens atletas (1) Mulheres diagnosticadas com a forma clinica de remitente- recorrente de esclerose múltipla (1), Familiares de pacientes com Insuficiência Cardíaca (1) Pacientes com Doença de Parkinson (1) Pessoas com Síndrome dolorosa pós laminectomia (1), Fumantes (1), Pacientes com implantes de stent coronário (1), Cuidadores de Crianças (1), Pacientes amputados de ambos os sexos (1) e Policiais militares, Adolescentes (1) Uma outra análise realizada a partir das produções cientificas recuperadas, atentou-se para os instrumentos que vem sendo utilizados para avaliação da ansiedade, bem como os construtos associados. A Tabela 3 mostra tais informações. 
Tabela 3. Construtos correlacionados com ansiedade e instrumentos utilizados para avaliação.

\begin{tabular}{clc}
\hline Construto & \multicolumn{1}{c}{ Instrumentos } & N \\
\hline Depressão & Inventario de Beck de Depressão & 29 \\
Desesperança & Inventário de Beck de Desesperança & 3 \\
Personalidade & Pfister & 3 \\
Relação mãe e feto & Escala de Apego Materno-Fetal e Escala de Modos & \\
& de Enfrentamento do Problema & 3 \\
Vínculo com os pares & Escala de Vinculo do Adulto & 1 \\
Transtorno de estresse pós- & Clinical Global Impression (CGI) e o PTSD & 1 \\
traumático (TEPT) & Checklist - civilianversion. & 1 \\
Sonolência diurna excessiva & Escala de Sonolência Epworth & 1 \\
Estresse psicológico & Inventário de sintomas de estresse de Lipp para \\
Compulsão Alimentar & adultos (ISSL) & 3 \\
Fibromialgia & Escala de Compulsão Alimentar Periódica. & 2 \\
Outras escalas & Questionário de impacto de fibromialgia & 7 \\
\hline
\end{tabular}

No que diz respeito aos construtos correlacionados com ansiedade e instrumentos utilizados para avaliação, atenta-se que a ansiedade vem sendo estudada em associação com depressão, desesperança, personalidade, neuroticismo, abuso de drogas, conduta antissocial, relação mãe e feto, vinculo com os pares, transtorno de estresse pós traumático, sonolência diurna, estresse psicológico, personalidade tipo $D$ e compulsão alimentar periódica. Dentre os construtos investigados viu-se prevalência na relação da ansiedade com a depressão (29), seguido da relação da ansiedade com a desesperança (3) e relação mãe e feto (2). Ainda foram encontradas associações com compulsão alimentar e fibromialgia,

Para a análise dos construtos os instrumentos utilizados foram: Inventário Beck de Depressão, Inventário Beck de Desesperança, Técnica Projetiva do desenho, casa, árvore e pessoa (H.T.P.), Pfister, Escala Fatorial de Ajustamento Emocional/Neuroticismo, Escala Analógico-visual, Fagerstrom Test For NicotineDependende, Mini-exame do Estado Mental, Escala de Apego Materno Fetal, Escala de Modos de Enfrentamento do Problema, Escala de Vinculação do Adulto, Clinical Global Impression (CGI), PTSD Checklist - civilianversion, Escala de Sonolência Epworth, Inventário de Sintomas de Estresse de Lipp para Adultos (ISSSL), Escala de Personalidade Tipo D e Escala de Compulsão Alimentar. E dentre os instrumentos usados houve uma prevalência das Escalas Beck de Depressão (15),seguido da Escala Beck de Desesperança (3), por último a Escala de Apego Materno Fetal (2) e a Escala de Modos de Enfrentamento do Problema (2).

\section{DISCUSSÃO}

Embora o presente estudo busque verificar a produção científica dos últimos dez anos em periódicos nacionais referentes à avaliação da ansiedade pode-se observar uma prevalência de associações da ansiedade com construtos diversos. Especificamente, verificou-se uma prevalência de estudos referente a associação entre ansiedade e depressão.

Segundo Marx et al (2011) a depressão e a ansiedade são doenças psiquiátricas comuns na população geral [...]. Ambas estão entre as doenças mentais crônicas mais prevalentes em todo o mundo. No Brasil, $15 \%$ a $25 \%$ dos estudantes universitários apresentam algum tipo de transtorno psiquiátrico durante sua formação acadêmica, consideravelmente transtornos depressivos e de ansiedade (NOGUEIRA, 2013).

Referente aos construtos correlacionados com ansiedade e instrumentos utilizados para avaliação houve uma prevalência na aplicação das Escalas Beck, principalmente a de 
Depressão devido a alta associação entre os construtos. A Escala Beck tem por finalidade uma rápida avaliação de estados de humor, sendo adequada para pacientes na faixa de 17 a 80 anos de idade, e pode ser usada em indivíduos sem transtornos psiquiátricos. E consiste de quatro escalas que mensuram sintomas de ansiedade, depressão, desesperança e ideação suicida, apresentando evidências de validade para a população adulta brasileira (OLIVEIRA et al, 2006).

Visto que a ansiedade é um construto que vem sendo amplamente estudado e encontram-se diversos artigos que abordam a associação deste com a depressão, Araújo, Nakano e Gouveia (2009) salientam que é importante destacar que ela faz parte dos sentimentos normais, tornando-se patológica quando a intensidade ou frequência não satisfaz à situação que a desencadeia ou quando não existe um objeto específico ao qual se direcione. Essa classificação vai depender da situação em si, das características do indivíduo e da interpretação que ele faz da situação.

Contudo o uso de instrumentos torna-se de grande importância para avaliar a intensidade e a frequência da ansiedade patológica presente na vida dos indivíduos, desta forma a Escala Beck mostra-se um instrumento eficaz e satisfatório para a prática desses profissionais.

\section{CONCLUSÃO}

Diante da revisão, os artigos sobre ansiedade abordam o tema como um dos transtornos mais comuns visto na população geral, porém, mostra-se pouco investigada em populações específicas como adolescentes, idosos e pacientes internados. Pode-se perceber que alguns contextos limitaram o estudo sendo direcionados mais para o ambiente educacional, âmbito que ficou evidente favorecer a ansiedade, deixando de analisar outros contextos diários que são significativos para o surgimento desse transtorno.

Os artigos analisados mostraram que houve prevalência em publicações sobre a ansiedade nas revistas de área médica, necessitando ampliação de estudos nas revistas específicas de Psicologia, assim como o aumento de estudos que validem a Escala Beck. Por fim, nota-se relevante compreender a ansiedade e suas implicações em diferentes contextos, bem como necessário dispor de instrumentos efeitos para a avaliação desse construto.

\section{REFERÊNCIAS}

ARAUJO, Fábio Alves Ferreira Maia de; NAKANO, Tatiana de Cássia; GOUVEIA, Maria Lígia de Aquino. Prevalência de depressão e ansiedade em detentos. Aval. psicol., Porto Alegre , v. 8, n. 3, p. 381-390, dez. 2009 . Disponível em <http://pepsic.bvsalud.org/scielo.php?script=sci_arttext\&pid=S1677-

04712009000300010\&lng=pt\&nrm=iso>. acessos em 28 jul. 2017.

BATISTA, Marco Antonio; OLIVEIRA, Sandra Sales. Sintomas de ansiedade mais comuns em adolescentes. PSIC - Revista de Psicologia da Vetor Editora, v. 6, no 2, p. 43-50, Jul./Dez. 2005

BEIGN, Gabriel de Azambuja et al . Impacto psicossocial e na qualidade de vida de pacientes com síndrome dolorosa após laminectomia. Rev. dor, São Paulo , v. 16, n. 1, p. 3236, mar. $2015 \quad$ Disponível em $<$ http://www.scielo.br/scielo.php?script=sci_arttext\&pid=S1806$00132015000100032 \&$ Ing=pt\&nrm=iso $>$. acessos em 25 maio 2017. 
GUERRA, Priscilla Caetano et al. Sono, qualidade de vida e humor em profissionais de enfermagem em Unidades de Terapia Intensiva Infantil. Rev. esc. enferm. USP, São Paulo , v. 50, n. 2, p. 279-285, abr. 2016 . Disponível em <http://www.scielo.br/scielo.php?script=sci_arttext\&pid=S008062342016000200279\&lng=pt\&nrm=iso >. acessos em 25 maio 2017.

LACERDA, Marianna Sobral et al . Ansiedade, estresse e depressão de familiares de pacientes com insuficiência cardíaca. Rev. esc. enferm. USP, São Paulo , v. 51, e03211, 2017 . Disponível em <http://www.scielo.br/scielo.php?script=sci_arttext\&pid=S008062342017000100403\&lng=pt\&nrm=iso>. acessos em 25 maio 2017.

MARX, C. et al . Depressão, ansiedade e sonolência diurna em cuidadores primários de crianças com paralisia cerebral. Rev. Paul. Pediatr. São Paulo , v. 29, n. 4, dez. 2011. Disponível em: <http://www.scielo.br/scielo.php?script=sci_arttext\&pid=S010305822011000400003\&lng=pt\&nrm=iso>. Acesso em: 03 nov. 2014.

NOGUEIRA, T. G. O teste de Pfister na avaliação de depressão e ansiedade em universitários: evidências preliminares. Bol. psicol, São Paulo, v. 63, n.

138, jun. 2013. Disponível em: <http://pepsic.bvsalud.org/scielo.php?script=sci_arttext\&pid=S000659432013000200003\&lng=pt\&nrm=iso>. Acesso em: 17 nov. 2014.

OLIVEIRA, K. L. de et al . Relação entre ansiedade, depressão e desesperança entre grupos de idosos. Psicol. Estud., Maringá, v. 11, n. 2, ago. 2006. Disponível em: $<$ http://www.scielo.br/scielo.php?script=sci_arttext\&pid=S141373722006000200014\&lng=p t\&nrm=iso>. Acesso em: 03 nov. 2014.

STALL, Paula et al . Efeitos do método Rolfing ${ }^{\circledR}$ de integração estrutural e da acupuntura na fibromialgia. Rev. dor, São Paulo , v. 16, n. 2, p. 96-101, jun. 2015 . Disponível em <http://www.scielo.br/scielo.php?script=sci_arttext\&pid=S180600132015000200096\&lng=pt\&nrm=iso>. acessos em 25 maio 2017.

TROCOLI, Tathiana O.; BOTELHO, Ricardo V.. Prevalência de ansiedade, depressão e cinesiofobia em pacientes com lombalgia e sua associação com os sintomas da lombalgia. Rev. Bras. Reumatol., São Paulo, v. 56, n. 4, p. 330-336, ago. 2016 . Disponível em <http://www.scielo.br/scielo.php?script=sci_arttext\&pid=S048250042016000400330\&lng=pt\&nrm=iso>. acessos em 25 maio 2017. 